\title{
The Application of Mathematical Modeling in Product Process Design
}

\author{
Qi Te \\ College of information science and Engineering \\ Central South University \\ Changsha, China \\ qite1030@163.com \\ Liu Chenqi \\ College of information science and Engineering \\ Central South University \\ Changsha, China
}

\author{
Fang Shangpeng \\ College of information science and Engineering \\ Central South University \\ Changsha, China
}

\begin{abstract}
As the improvement of living standard, people are more and more pursuing personalized furniture. For more and more personalized custom products, furniture production designers can no longer fix processing parameters by the means of test sample. There is an urgent need of precise parameter calculation to meet the design requirement in flow production process. Taking a creative folding table as an example, this paper studies how to use mathematical modeling and programming calculation method in the design of personalized products. In this paper, the surface equation of folding table legs is given by the vector algebra method. The mathematical model for calculating the processing parameters of folding table leg is set up. By Matlab programming the dynamic changing process of the edge curve of table legs is drawn, and the main parameters of slot on the table legs are calculated.
\end{abstract}

Keywords-Mathematical modeling;Straight grain surface; Parametric equations; processing technology; Matlab

\section{INTRODUCTION}

Wood products production belongs to the traditional handicraft industry; its processing technology relies on the wood with exquisite workmanship and rich experience, in order to achieve the purpose of the firm and beautiful. With the popularity of woodworking machinery, the furniture of a few simple structures, model specification products basically achieved components batch processing, and use the site field assembly line production. Product of this mechanized production, are generally by simple calculation, trial production, the actual measurement process to determine the processing parameters such as sample. However, because of the unequal wood shrinkage, easy to deformation, etc, in order to assemble smoothly, its parts processing parameters are greater leeway, its slitting, punching size is usually thought to be the key to the traditional process parameters, such as amplification, assembly when rely on components such as special screw fixation.

As the improvement of living standard, people more and more personalized pursuit on furniture and other products. For more and more personalized custom products, furniture production enterprises could no longer use the method of test sample processing parameters, an urgent need to precise calculation to meet the design requirements of batch production process. In a creative folding table, for example, this paper studies how to use mathematical modeling ${ }^{[1-4]}$ and programming calculation ${ }^{[5]}$ method, product process design, in order to meet the demand for personalized products.

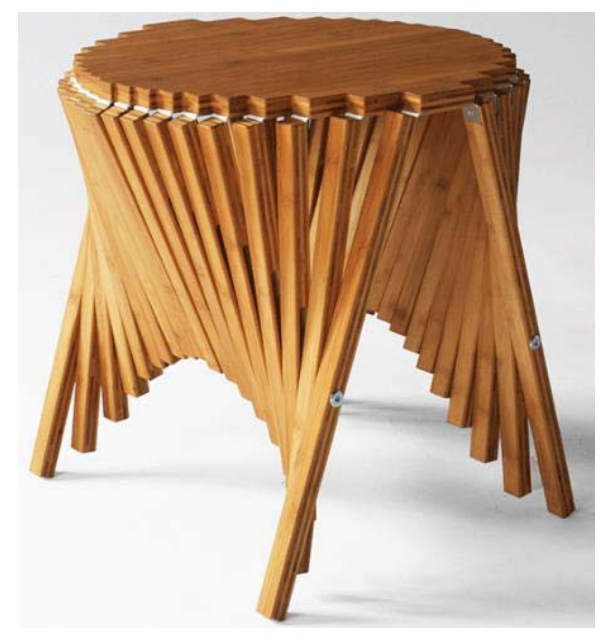

Figure 1. Circular folding table

In Fig.1, the company in the production of a folding table, desktop assumes the circular, table legs as the hinge of the activity can be split into a rectangular plate. Table leg consists of a number of sticks, divided into two groups, each group with a reinforced connect wood, respectively fixed on both ends of the steel in the table legs each two sticks on the outside piece, and along the wood's free slots to ensure the freedom of sliding. Table consists of ruled surface appearance, beautiful appearance. Company plans to develop a folding table design software, according to the 
customer set arbitrary folding table height, and the size of the desktop and legs Angle, the shape of plate material required size and practical optimal design and processing parameters, makes the production of folding table as close as possible to the customer the desired shape.

\section{MATHEMAtical Model OF Folding TABLE LeG SURFACE}

In order to facilitate research, assuming the width and thickness are $r, s$ respectively, desktop radius is $R$, height is $h$, the angle between the lateral leg of table and a horizontal surface is $\varphi$, the distance of between the steel position of outer table leg and the top of table leg is $p$, the same side of the outer table leg end-to-end distance is $2 d$, the rectangular plate length is $2 L$.

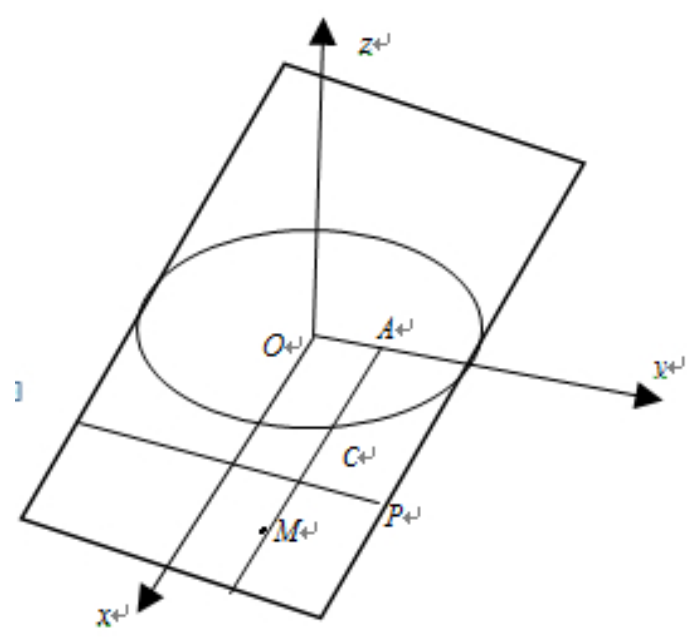

Figure 2. Folding table before folding diagram

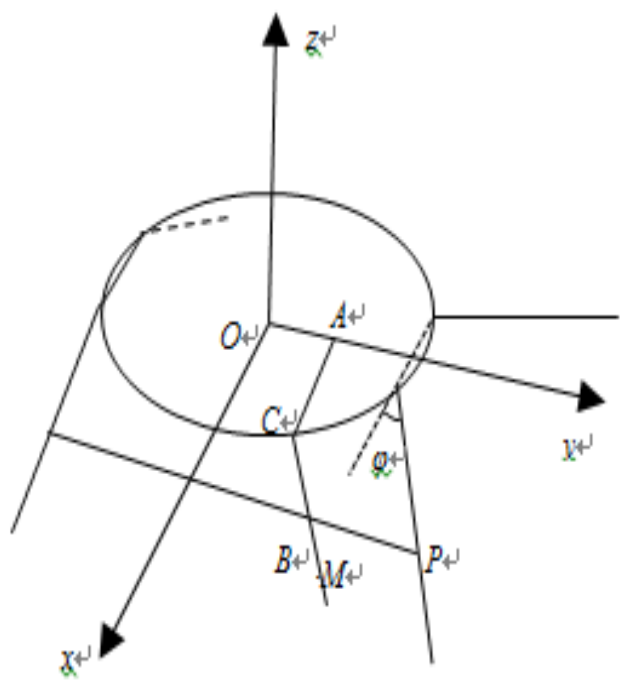

Figure 3. Folding table after folding diagram

As shown in Fig.3, establish coordinate system, where the $x$ axis is parallel to the direction of the desktop of wood. For any fixed $\varphi$, the following We derive the equation of table leg surface and its edge curve. According to the symmetry, we only consider the $x$ axis pointing to the side.

\section{A. Straight Grain Surface Equation of Table Legs}

Let $M$ be any point of a table leg, then we calculate vector $\overrightarrow{O M}$. As shown in fig.2, The angle between $\overrightarrow{O C}$ and the $\mathrm{x}$ axis is $\theta(-\pi / 2 \leq \theta \leq \pi / 2), \quad P$ is the intersection outermost table legs and reinforced. Before folding $M$ to $y$ axis distance is $u$. Then equation of the edge of desktop be:

$$
\left\{\begin{array}{l}
x=R \cos \theta \\
y=R \sin \theta,-\pi<\theta \leq \pi \\
z=0
\end{array}\right.
$$

Here, we use the formula

$$
\overrightarrow{O M}=\overrightarrow{O C}+\overrightarrow{C M}
$$

to calculate table leg lateral ruled surface parameter equation.

Due to the $\mathrm{C}$ point on the circumference of a circle, so

$$
\overrightarrow{O C}=(R \cos \theta, R \sin \theta, 0) \text {. }
$$

Let the coordinate of $B, P$ are

$$
\left(x_{B}, R \cos \theta, z_{B}\right) \text { and }\left(x_{P}, R, z_{P}\right) \text {. }
$$

Because of the straight line $B P$ parallel to the $y$ axis, so

$$
\begin{aligned}
& x_{B}=x_{P}=p \cos \varphi+d \\
& z_{B}=z_{P}=-p \sin \varphi
\end{aligned}
$$

then

$$
\begin{aligned}
& \overrightarrow{C B}=(p \cos \varphi-R \sin \theta+d, 0,-p \sin \varphi) \\
& |\overrightarrow{C B}|=\sqrt{(p \cos \varphi-R \sin \theta+d)^{2}+p^{2} \sin ^{2} \varphi}
\end{aligned}
$$

Because

$$
|\overrightarrow{C M}|=u-R \sin \theta
$$

Therefore,

$$
\begin{aligned}
\overrightarrow{C M} & =|\overrightarrow{C M}| \cdot \frac{\overrightarrow{C B}}{|\overrightarrow{C B}|} \\
& =\frac{(u-R \sin \theta)(p \cos \varphi-R \sin \theta+d, 0,-p \sin \varphi)}{\sqrt{(p \cos \varphi-R \sin \theta+d)^{2}+p^{2} \sin ^{2} \varphi}}
\end{aligned}
$$

Because

$$
\overrightarrow{O M}=\overrightarrow{O C}+\overrightarrow{C M}
$$

So

$$
\begin{aligned}
\overrightarrow{O M} & =\overrightarrow{O C}+\overrightarrow{C M} \\
& =\left\{R \sin \theta+\frac{(u-R \sin \theta)(p \cos \alpha-R \sin \theta+d)}{\sqrt{(p \cos \alpha-R \sin \theta+r)^{2}+p^{2} \sin ^{2} \alpha}},\right. \\
& \left.R \cos \theta,-\frac{(u-R \sin \theta) p \sin \alpha}{\sqrt{(p \cos \alpha-R \sin \theta+d)^{2}+p^{2} \sin ^{2} \alpha}}\right\}
\end{aligned}
$$

Then, we get table leg lateral ruled surface parameter equation: 


$$
\left\{\begin{array}{l}
x=R \sin \theta+\frac{(u-R \sin \theta)(p \cos \varphi-R \sin \theta+d)}{\sqrt{(p \cos \varphi-R \sin \theta+d)^{2}+p^{2} \sin ^{2} \varphi}}, \\
y=R \cos \theta, \\
z=-\frac{(u-R \sin \theta) p \sin \varphi}{\sqrt{(p \cos \varphi-R \sin \theta+d)^{2}+p^{2} \sin ^{2} \varphi}}, \\
0 \leq u \leq L,-\frac{\pi}{2} \leq \theta \leq \frac{\pi}{2}
\end{array}\right.
$$

Elimination parameters $u, \theta$, we get:

$$
(z-p \sin \varphi) \sqrt{R^{2}-y^{2}}=p(x \sin \varphi-z \cos \varphi)+d z
$$

When the demand $\varphi$ is gradually increasing from 0 to tuck up the biggest angle $\alpha$, we get the dynamic changes of the folding table in the process of dynamic change process.

\section{B. Curve Equation of Edge of the Table Legs}

In the table leg lateral surface parameter equation, the parameters of the corresponding points on the edge of the table leg is L. Therefore, we can get the table leg edge curve parameter equation by replace $u$ with $L$.

$$
\left\{\begin{array}{l}
x=R \sin \theta+\frac{(L-R \sin \theta)(p \cos \varphi-R \sin \theta+d)}{\sqrt{(p \cos \varphi-R \sin \theta+d)^{2}+p^{2} \sin ^{2} \varphi}}, \\
y=R \cos \theta, \\
z=-\frac{(L-R \sin \theta) p \sin \varphi}{\sqrt{(p \cos \varphi-R \sin \theta+d)^{2}+p^{2} \sin ^{2} \varphi}}, \\
-\frac{\pi}{2} \leq \theta \leq \frac{\pi}{2}
\end{array}\right.
$$

Elimination parameters $\theta$, and tidy get curve equation of edge of the table legs:

$$
\left\{\begin{array}{l}
(z-p \sin \varphi) \sqrt{R^{2}-y^{2}}=p(x \sin \varphi-z \cos \varphi)+d z \\
z^{2}\left[L-\sqrt{R^{2}-y^{2}}\right]=p\left[\left(L-\sqrt{R^{2}-y^{2}}\right)^{2}-z^{2}\right] \sin \varphi
\end{array}\right.
$$

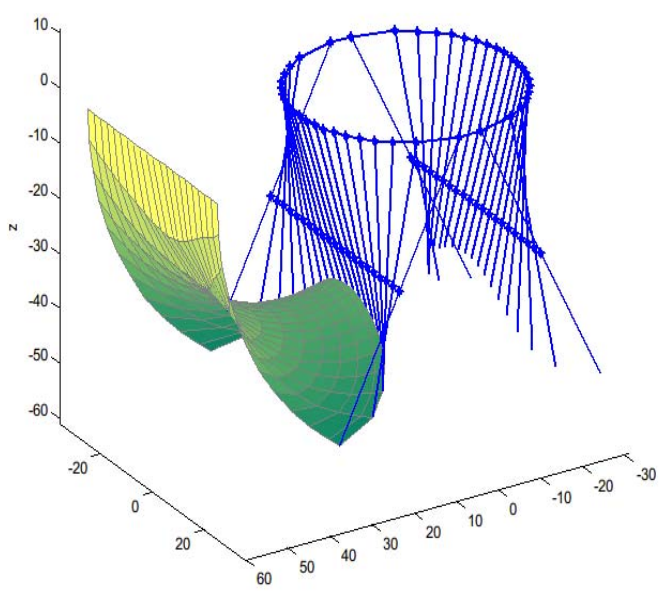

Figure 4. Table leg edge in the folding process change
Let

$$
L=60, R=50, r=d=2.5, p=28.75,
$$

use Matlab ${ }^{[6,7]}$ to draw out under the table leg edge swept surface as shown in Fig.4.

\section{Folding TABle Processing Parameters}

\section{A. Mathematical Model OF Processing Parameters}

Assume that users will need to order circular folding table. The desktop radius is $R$, the higher is $h$. Wood specifications is $2 L \times r \times s$, need number for wood is $2 n$, here $n=\lfloor R / r\rfloor$. Below, we calculation the slot parameters of each table leg by mathematical modeling.

According to the symmetry, we consider only in $\mathrm{V}$ octant in fig. 2. The table legs are numbered from left to right, on behalf of the leg of the table with a table leg right inside line, then the top corresponding parameters $\theta$ of $\mathrm{n}^{\text {th }}$ legs satisfy:

$$
R \sin \theta=\frac{r i}{R}
$$

Let the two endpoints of table leg slot be $E_{i}, F_{i}$ respectively, $E_{i}, F_{i}$ point is essentially steel bar location before and after folding. Let

$$
\begin{aligned}
& l_{i}=C_{i} E_{i}, \\
& l_{i}{ }^{\prime}=C_{i} F_{i},
\end{aligned}
$$

then the upper and lower position of slot are:

$$
\begin{gathered}
l_{i}=p+d-R \sin \theta \\
=p+d-\frac{r i}{R} \\
\overrightarrow{C_{i} F_{i}}=\left\{p \cos \alpha+d-\frac{r i}{R}, 0,-p \sin \alpha\right\},
\end{gathered}
$$

So, the lower end point of slot are:

$$
\begin{aligned}
l_{i}{ }^{\prime} & =\left|\overrightarrow{C_{i} F_{i}}\right| \\
& =\sqrt{\left(p \cos \alpha+d-\frac{r i}{R}\right)^{2}+p^{2} \sin ^{2} \alpha}
\end{aligned}
$$

The slot length $i^{\text {th }}(i=1,2, \cdots, n)$ table leg is

$$
\begin{aligned}
S & =l_{i}{ }^{\prime}-l_{i}{ }^{\prime} \\
& =\sqrt{\left(p \cos \alpha+d-\frac{r i}{R}\right)^{2}+p^{2} \sin ^{2} \alpha}-\left(p+d-\frac{r i}{R}\right)
\end{aligned}
$$

\section{B. The Practical Application}

For example, with size of $120 \times 50 \times 3 \mathrm{~cm}$ rectangle plate processed into high $h=53 \mathrm{~cm}$ round folding table. Namely $L=60 \mathrm{~cm}, r=2.5 \mathrm{~cm}, s=3, n=10$. Take two longest length of sticks for desktop is $50 \mathrm{~cm}, d=r$, so the desktop circumscribed circle radius is 


$$
R=\sqrt{25^{2}+2.5^{2}}=25.125
$$

Suppose the steel fixed in the central position of the outer side leg of the table, so

$$
\begin{aligned}
& p=\frac{L-r}{2}=\frac{60-2.5}{2}=28.75 \\
& \sin \alpha=\frac{h-s}{L-r}=\frac{50}{57.5} \approx 0.26, \\
& \alpha \approx 1.054 \approx 60.4^{\circ}
\end{aligned}
$$

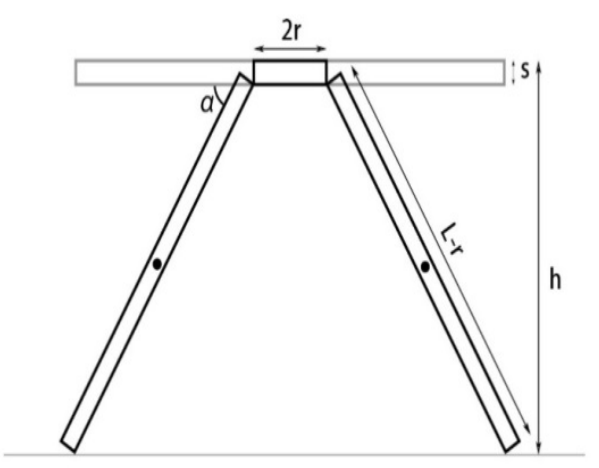

Figure 5. Folding table view

The above parameters generation into the slot parameter calculation formula, we get the table legs batten slot parameters:

\section{TABLE I. TABLE LEGS BATTEN SLOT PARAMETERS}

\begin{tabular}{|c|c|c|c|c|c|c|c|c|c|c|}
\hline Num. & $\mathbf{1}$ & $\mathbf{2}$ & $\mathbf{3}$ & $\mathbf{4}$ & $\mathbf{5}$ & $\mathbf{6}$ & $\mathbf{7}$ & $\mathbf{8}$ & $\mathbf{9}$ & $\mathbf{1 0}$ \\
\hline Top & 6.3 & 6.6 & 7.3 & 8.2 & 9.5 & 11.1 & 13.2 & 16.0 & 20.1 & 28.8 \\
\hline End & 26.3 & 26.2 & 26.0 & 25.8 & 25.5 & 25.2 & 25.0 & 25.0 & 25.6 & 28.8 \\
\hline Length & 20.1 & 19.6 & 18.8 & 17.6 & 16.1 & 14.2 & 11.8 & 9.0 & 5.5 & 0.0 \\
\hline
\end{tabular}

\section{THE CONCLUSION}

With the continuous improvement of people's living standard, people for the personalized pursuit of creative products are higher and higher. It is moss-grown that through the mass production to reduce the production cost model already, customize product according to user needs become a trend. How to reduce the cost for small batch products in the design, manufacture, become the focus. Its main method there are $\mathrm{two}^{[8-10]}$.The first method is to save the cost, design and computer aided design the first method is to mathematical modeling to saving the cost of manufacture. In this paper, through mathematical modeling and supplemented by computer programming calculation, accurate product processing process parameters, reduce the design costs, such as test samples, can achieve precise and quick.

\section{REFERENCES}

[1] Han Zhonggen. Mathematical modeling method and its application. Beijing: Higher education press, 2008.

[2] Jiang Qiyuan, Xie Jinxin, Ye Jun. Mathematical model. Beijing: Higher education press, 2011.

[3] Tan Tongjun, Cai Zhijie. Mathematical model. Shanghai: Fudan university press, 2011.

[4] Si Shoukui, Sun Xiqing. Mathematical modeling algorithm and application. National defence industry press, 2011.

[5] Liu Weiguo. MATLAB programming tutorial. China water conservancy and hydropower press, 2009.

[6] Zhuo Jinwu. The application of MATLAB in mathematical modeling. Beijing aerospace university press, 2011.

[7] Le Jinliang, Xiang Longwan, Li Shidong. Mathematics experiment. Higher education press, 2011.

[8] Liu Jianfeng, Sun Yazhou, Lu Zesheng. Micro-milling Parameter Optimization Based on Genetic Algorithm. Aviation Precision Manufacturing Technology,2010,46(5): 23-26.

[9] Hwang ShunFa. Improv ing Real- param eter Genetic Algo rithm with Simulated Annealing for Engineering Problems. Advances in Engineering Software 2006, 37: 406 418

[10] G. Ioannis. Solving Constrained Optimization Problems Using a Novel Genetic Algorithm. App lied Mathematics and Computation, 2009, 208: 273 283 